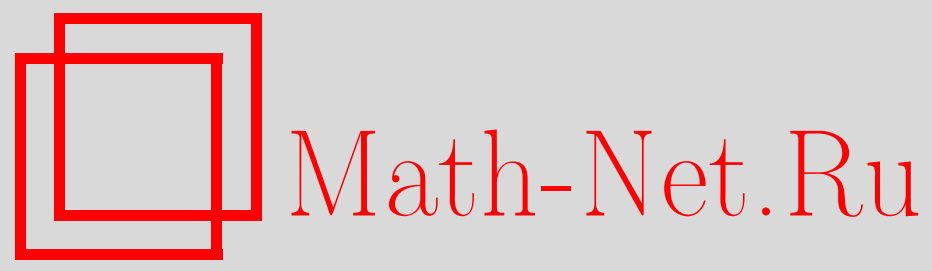

А. В. Бабаш, Автоматные отображения слов, размножающие искажения в метриках Хемминга и Левенштейна не более, чем в $K$ раз, Дискрет. матем., 2002, том 14, выпуск $3,78-94$

DOI: https://doi.org/10.4213/dm256

Использование Общероссийского математического портала Math-Net.Ru подразумевает, что вы прочитали и согласны с пользовательским соглашением http://www.mathnet.ru/rus/agreement

Параметры загрузки:

IP : 107.22 .136 .117

26 апреля 2023 г., 16:41:55 


\title{
Автоматные отображения слов, размножающие искажения в метриках Хемминга и Левенштейна не более, чем B $K$ pa3
}

\author{
(c) 2002 г. $\quad$ A. В. Бабаш
}

\begin{abstract}
Пусть $I$ и $O$ - конечные алффавиты. Для конечного алфавита $\Omega$ будем обозначать через $\Omega^{*}$ множество всех слов конечных длин в алфавите $\Omega$. В статье дается полное описание всех автоматных отображений множества $I^{*}$ в $O^{*}$, которые размножают ошибки типа замены букв в словах не более, чем в $K$ раз. Дается полное описание инъективных автоматных отображений множества $I^{*}$ в $O^{*}$, которые размножают ошибки типа пропуска букв не более, чем в $K$ раз. Аналогичный результат получен для метрики выпадений и вставок букв.
\end{abstract}

\section{1. Введение}

Пусть $I^{*}, O^{*}$ и $\Omega^{*}$ - множества всех слов конечных длин в конечных алфавитах $I, O$ и $\Omega$ соответственно. В работе продолжаются исследования, связанные с проблемой описания отображений множества $I^{*}$ в множество $O^{*}$, которые размножают ошибки типа замены букв не более, чем в $K$ раз. Данная проблема была поставлена А. А. Марковым в 1956 году. Началом исследований явилась неопубликованная работа А. А. Маркова, о которой сообцалось в [1]. Им были описаны все биективные преобразования множества слов $\Omega^{*}$, сохраняющие длины слов и не увеличивающие расстояния Хемминга между словами одной длины, то есть не размножающие искажения типа замены букв в словах. Дальнейшие исследования по указанной проблеме, связанные с исследованием инъективных отображений, а также с рассмотрением и других искажений слов, например, искажений типа выпадений или вставки букв в слова, были проведены в [1]. Описание же всех инъективных отображений множества $I^{*}$ в $O^{*}$, не размножающих ошибки типа пропуска букв, было дано в [2]. Таким образом, говоря о конечных результатах исследований по указанной проблеме А. А. Маркова, можно сказать, что она решена пока в частном случае $K=1$, при этом описаны лишь биективные отображения с требуемым свойством. В данной работе дается полное описание произвольных (то есть возможно не инъективных) конечно-автоматных отображений множества $I^{*}$ в $O^{*}$, которые размножают ошибки типа замены букв не более, чем в $K$ раз. Дается и полное описание инъективных конечноавтоматных отображений множества $I^{*}$ в $O^{*}$, которые размножают ошибки типа пропуска букв не более, чем в $K$ раз. Аналогичный результат получен для метрики выпадений и вставок букв (см. [3]). 


\section{2. Обозначения и основные понятия}

Будем использовать следующие обозначения. Пусть $|\Omega|-$ мощность множества $\Omega ; I, O$ - конечные алфавиты $|I|>1,|O|>1 ; I^{*}$ и $O^{*}$ - множества всех слов конечной длиньі алфавитов $I$ и $O$, соответственно, считаем, что пустое слово $\varnothing$ принадлежит $I^{*}$ и $O^{*}$; для слов $\mathfrak{\Im}, \mathfrak{I}^{\prime} \in I^{k}, \mathfrak{I}=i_{1} \ldots i_{k}, \mathfrak{I}^{\prime}=i_{1}^{\prime} \ldots i_{k}^{\prime}$, будем обозначать $\rho\left(\mathfrak{s}, \mathfrak{\Im}^{\prime}\right)$ расстояние Хемминга между словами $\mathfrak{s}, \mathfrak{\Im}^{\prime} \in I^{k}$.

Определение 1. Отображение $\varphi: I^{*} \rightarrow O^{*}$ называется отображением, увеличивающим метрику $\rho$ не более, чем в $K$ раз, если для любых слов $\mathfrak{s}, \mathfrak{\Im}^{\prime} \in I^{*}$ выполняются условия

$$
\begin{gathered}
|\mathfrak{s}|=\left|\mathfrak{I}^{\prime}\right| \Longrightarrow|\varphi(\mathfrak{s})|=\left|\varphi\left(\mathfrak{I}^{\prime}\right)\right|, \\
K \rho\left(\mathfrak{s}, \mathfrak{\Im}^{\prime}\right) \geqslant \rho\left(\varphi(\mathfrak{s}), \varphi\left(\mathfrak{\Im}^{\prime}\right)\right) .
\end{gathered}
$$

При $K=1$ такое отображение называют не увеличивающим метрику $\rho$ (не размножающим искажения типа замены букв [1]).

Множество всех отображений $\varphi: I^{*} \rightarrow O^{*}$, увеличивающих метрику $\rho$ не более, чем в $K$ раз, обозначим через $G\left(I^{*}, O^{*}, \rho, K\right)$. Множество всех инъективных отображений из $G\left(I^{*}, O^{*}, \rho, 1\right)$ полностью описано в [1].

Через $A=\left(I, S, O,\left(\delta_{i}\right)_{i \in I},\left(\beta_{i}\right)_{i \in I}\right)$ обозначим конечный автомат с входным алфавитом $I$, множеством состояний $S$, выходным алфавитом $O$, частичными функщиями переходов $\delta_{i}: S \rightarrow S, i \in I$, и частичными функциями выхода $\beta_{i}: S \rightarrow S, i \in I$. Выходное слово автомата $A$, соответствующее входному слову $\mathfrak{s} \in I^{*}$ и начальному состоянию $s \in S$ обозначим через $A(s, \Im)$; при $\mathfrak{s}=i_{1} \ldots i_{k}$ слово

$$
A(s, \Im)=y_{i} \ldots y_{k}=\beta_{i 1} s \beta_{i_{2}} \delta_{i_{1}} s \ldots \beta_{i_{k}} \delta_{i_{k-1}} \ldots \delta_{i_{1}} s, \quad i_{j} \in I, \quad y_{j} \in O, \quad j=1, \ldots, k .
$$

Автоматным отображением назовем отображение $A_{s}: I^{*} \rightarrow O^{*}$, задаваемое равенством $A_{s}(\mathfrak{\Im})=A(s, \mathfrak{I})$. Для описания автоматного отображения $A_{s}$ будем использовать подавтомат $A[s]$ автомата $A$, порожденный состоянием $s \in S$. Множеством состояний автомата $A[s]$ является подмножество $S_{s}$ множества $S$, состоящее из состояния $s$ и всех состояний, достижимых из $s$ в графе переходов автомата $A$. Подграф $\Gamma_{s}$ графа автомата $A$, соответствующий множеству $S_{s}$, определяет подавтомат

$$
A[s]=\left(I, S_{s}, O,\left(\delta_{i}\right)_{i \in I},\left(\beta_{i}\right)_{i \in I}\right)
$$

автомата $A$. Здесь для простоты обозначений ограничения отображений $\left(\delta_{i}\right)_{i \in I}$ и $\left(\beta_{i}\right)_{i \in I}$ на множество $S_{s}$ обозначены теми же символами.

Через $A\left(\left(I^{*}, O^{*}, \rho, K\right)\right.$ обозначим множество всех автоматных отображений

$$
A_{s}: I^{*} \rightarrow O^{*}
$$

увеличивающих метрику $\rho$ не более, чем в $K$ раз, то есть отображений $A_{s}$, для которых

$$
K \rho\left(\mathfrak{S}, \mathfrak{\Im}^{\prime}\right) \geqslant \rho\left(A_{s}(\mathfrak{S}), A_{s}\left(\mathfrak{S}^{\prime}\right)\right)
$$

при любых словах $\mathfrak{\Im}, \mathfrak{\Im}^{\prime} \in I^{*}$ одинаковой длины $\left(A_{s}(\varnothing)=\varnothing\right)$.

Полное описание множества $A\left(I^{*}, O^{*}, \rho, K\right)$ дано в разделе 3.

Обозначим через $\varepsilon$ бинарное отношение на множестве $I^{*}$. Запись $\mathfrak{\Im} \varepsilon \mathfrak{\Im}^{\prime}$ означает, что слово $\mathfrak{\Im}^{\prime}$ получено из $\mathfrak{\Im}$ удалением одного вхождения некоторой его буквы (є -- 
бинарное отношение выпадения буквы); $\varepsilon^{t}-t$-я степень бинарного отношения $\varepsilon$. Через $\varepsilon^{-1}$ обозначим обратное к $\varepsilon$ бинарное отношение, именно,

$$
\mathfrak{\Im}^{\prime} \varepsilon^{-1} \mathfrak{\Im}^{\prime} \Longleftrightarrow \mathfrak{I} \varepsilon \mathfrak{\Im}^{\prime},
$$

$\varepsilon^{-1}$ есть бинарное отношение вставки буквы.

Бинарное отношение $\varepsilon$ будет иметь тот же смысл и на множестве слов $O^{*}$.

Определение 2. Отображение $\varphi: I^{*} \rightarrow O^{*}$ называется отображением, увеличивающим бинарное отношение $\varepsilon$ не более, чем в $K$ раз, если для любых слов $\Im, \mathfrak{\Im}^{\prime} \in I^{*}$ и любого $n=1, \ldots,|\Im|$ найдется число $m \in\{1, \ldots, K n\}$ такое, что выполняется импликация

$$
\mathfrak{\Im} \varepsilon^{n} \mathfrak{\Im}^{\prime} \Longrightarrow \varphi(\mathfrak{I}) \varepsilon^{m} \varphi\left(\mathfrak{\Im}^{\prime}\right) \text {. }
$$

При $K=1$ такое отображение $\varphi$ будем называть не размножающим искажений типа пропуска букв (см. [1]).

Через $A G\left(I^{*}, O^{*}, \varepsilon, K\right)$ обозначим множество всех автоматных инъективных отображений $A_{s}: I^{*} \rightarrow O^{*}$, увеличивающих бинарное отношение не более, чем в $K$ раз. Описание множества $A G\left(I^{*}, O^{*}, \varepsilon, K\right)$ дается в разделе 4 .

Обозначим через $D$ метрику Левенштейна на множестве $I^{*}\left(O^{*}\right)$ (см. [3]); $D\left(\mathfrak{I}, \mathfrak{\Im}^{\prime}\right)$ означает минимальное число выпадений и вставок букв, требуемое для получения из слова $\Im$ слова $\Im^{\prime}$.

Определение 3. Отображение $\varphi: I^{*} \rightarrow O^{*}$ называется отображением, увеличивающим метрику $D$ не более, чем в $K$ раз, если для любых слов $\Im, \Im^{\prime} \in I^{*}$ выполняется условие

$$
K D\left(\mathfrak{I}, \mathfrak{\Im}^{\prime}\right) \geqslant D\left(\varphi(\mathfrak{\Im}), \varphi\left(\mathfrak{\Im}^{\prime}\right)\right)
$$

Через $A G\left(I^{*}, O^{*}, D, K\right)$ обозначим множество всех автоматных инъективных отображений $A_{s}: I^{*} \rightarrow O^{*}$, увеличивающих метрику $D$ не более, чем в $K$ раз. Описание множества $A G\left(I^{*}, O^{*}, D, K\right)$ дается в разделе 5 .

\section{3. Описание множества $A\left(I^{*}, O^{*}, \rho, K\right)$}

Определение 4. $B$-шестеркой автомата $A=\left(I, S, O,\left(\delta_{i}\right)_{i \in I},\left(\beta_{i}\right)_{i \in I}\right)$ называется набор

$$
\left(s, i, i^{\prime}, y, s_{1}, s_{1}^{\prime}\right) \in S \times I \times I \times O \times S \times S,
$$

для компонент которого выполняются условия

$$
\delta_{i} s=s_{1}, \quad \delta_{i^{\prime}} s=s_{1}^{\prime}, \quad s_{1} \neq s_{1}^{\prime}, \quad \beta_{i} s=\beta_{i^{\prime}} s=y, \quad i \neq i^{\prime} .
$$

$B$-шестерке $\left(s, i, i^{\prime}, y, s_{1}, s_{1}^{\prime}\right)$ в графе переходов автомата $A$ соответствует фрагмент

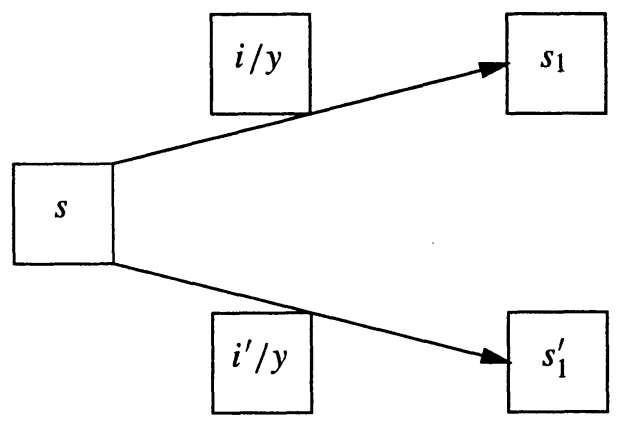


Для $\Im \in I^{k}, \mathfrak{\Im}=i_{1} i_{2} \ldots i_{k}$ положим $\delta_{\Im}=\delta_{i_{k}} \ldots \delta_{i_{2}} \delta_{i_{1}}$ и $\delta_{\varnothing} s=s$ при любом $s \in S$.

Определение 5. Приемником $B$-шестерки $\left(s, i, i^{\prime}, y, s_{1}, s_{1}^{\prime}\right)$ автомата $A$ называется тройка $\left(i^{\prime \prime}, s_{L}, s_{L}^{\prime}\right) \in I \times S \times S$, для элементов которой выполняются условия

- либо $s_{L}=s_{1}, s_{L}^{\prime}=s_{1}^{\prime}$ и $\beta_{i^{\prime \prime}} s_{L} \neq \beta_{i^{\prime \prime}} s_{L}^{\prime}$,

- либо $s_{L}=\delta_{\Im} s_{1}, s_{L}^{\prime}=\delta \Im s_{1}^{\prime}$ для некоторого $\Im \in I^{*} \backslash \varnothing$ и $\beta_{i^{\prime \prime}} s_{L}^{\prime} \neq \beta_{i^{\prime \prime}} s_{L}^{\prime}$.

При этом приемник $\left(i^{\prime \prime}, s_{L}, s_{L}^{\prime}\right)$ называется особым, если $\delta_{i^{\prime \prime}} s_{L}=\delta_{i^{\prime \prime}} s_{L}^{\prime}$; в противном случае приемник $\left(i^{\prime \prime}, s_{L}, s_{L}^{\prime}\right)$ называется неособым.

$B$-шестерке и ее приемнику в графе переходов автомата $A$ соответствует фрагмент

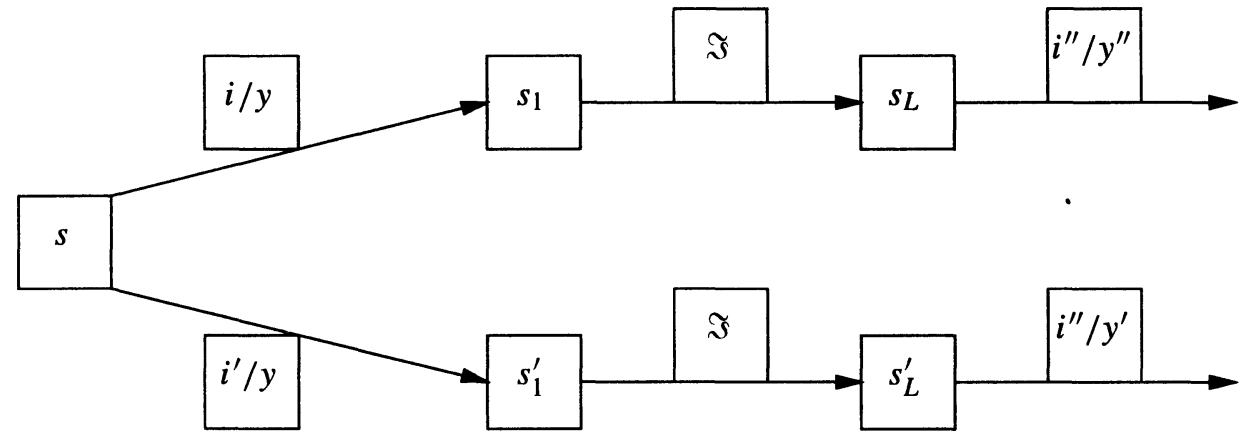

Определение 6. $B$-семеркой автомата $A$ называется набор

$$
\left(s, i, i^{\prime}, y, y^{\prime}, s_{1}, s_{1}^{\prime}\right) \in S \times I \times I \times O \times O \times S \times S,
$$

для компонент которого выполняются условия

$$
i \neq i^{\prime}, \quad y \neq y^{\prime}, \quad \delta_{i} s=s_{1}, \quad \delta_{i^{\prime}} s=s_{1}^{\prime}, \quad s_{1} \neq s_{1}^{\prime}, \quad \beta_{i} s=y, \quad \beta_{i} s=y^{\prime} .
$$

$B$-семерке автомата $A$ в его графе переходов соответствует фрагмент

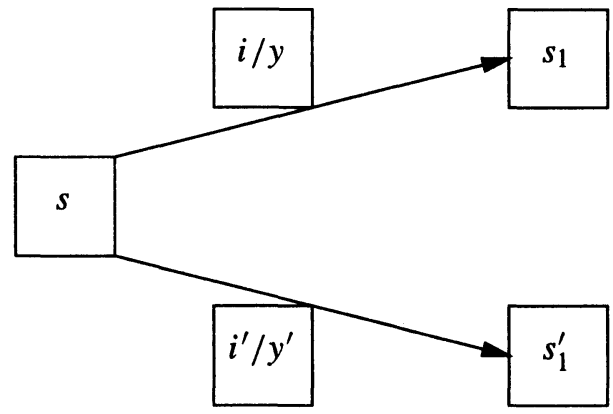

При описании множества $A\left(I^{*}, O^{*}, \rho, K\right)$ рассмотрим два случая $K=1$ и $K \geqslant 2$, которым соответствуют множество $A\left(I^{*}, O^{*}, \rho, K=1\right)$ всех автоматных отображений $I^{*}$ в $O^{*}$, не увеличивающих метрику $\rho$, и множество $A\left(\left(I^{*}, O^{*} \rho, K \geqslant 2\right)\right.$ автоматных отображений $I^{*}$ в $O^{*}$, увеличивающих метрику не более, чем в $K$ раз, $K \geqslant 2$.

Определение 7. Автомат $A$ называется внутренне автономным автоматом, если для любого состояния $s \in S$ и любых $i, i^{\prime} \in I$ справедливо равенство $\delta_{i} s=\delta_{i^{\prime}} s$. 
Согласно определению, переходы состояний внутренне автономного автомата не зависят от входного слова:

$$
\delta_{\Im} s=\delta_{\Im} s, s \in S .
$$

Напомним, что автомат $A=\left(I, S, O,\left(\delta_{i}\right)_{i \in I},\left(\beta_{i}\right)_{i \in I}\right)$ называется приведенным, если для любых различных состояний $s, s^{\prime} \in S$ найдется входное слово $\Im \in I^{*}$, при котором $A(s, \mathfrak{\Im}) \neq A\left(s^{\prime}, \mathfrak{\Im}\right)$.

Описание первого множества $A\left(I^{*}, O^{*}, \rho, K=1\right)$ дается следующей теоремой.

Теорема 1. Автоматное отображение $A_{s_{0}}$ не увеличивает метрику $\rho$ тогда и только тогда, когда в приведенном автомате

$$
A\left[s_{0}\right]=\left(I, S_{s_{0}}, O,\left(\delta_{i}\right)_{i \in I},\left(\beta_{i}\right)_{i \in I}\right),
$$

реализующем отображение $A_{s_{0}}$, во-первых, отсутствуют $B$-семерки и, во-вторых, либо автомат $A\left[s_{0}\right]$ является внутренне автономным автоматом, либо каждый из приемников любой В-шестерки автомата является особым.

Для описания множества $A\left(I^{*}, O^{*}, \rho, K \geqslant 2\right)$ введем дополнительные определения.

Определение 8. $K$-м приемником $(K \geqslant 1) B$-шестерки $\left(s, i_{1}, i_{1}^{\prime}, y, s_{1}, s_{1}^{\prime}\right)$ автомата $A$ $\left(B\right.$-семерки $\left.\left(s, i_{1}, i_{1}^{\prime}, y, y^{\prime}, s_{1}, s_{1}^{\prime}\right)\right)$ называется тройка $\left(i, s_{L}, s_{L}^{\prime}\right)$, компоненты которой удовлетворяют условиям $s_{L}=\delta_{\Im} s_{1}, \quad s_{L}^{\prime}=\delta_{\Im} s_{1}^{\prime}$ для некоторого слова $\Im \in I^{*}, \beta_{i} s_{L} \neq \beta_{i} s_{L}^{\prime}$

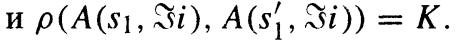

$B$-шестерке $\left(s, i_{1}, i_{1}^{\prime}, y, s_{1}, s_{1}^{\prime}\right)$ и ее $K$-му приемнику в графе переходов автомата $A$ соответствует фрагмент

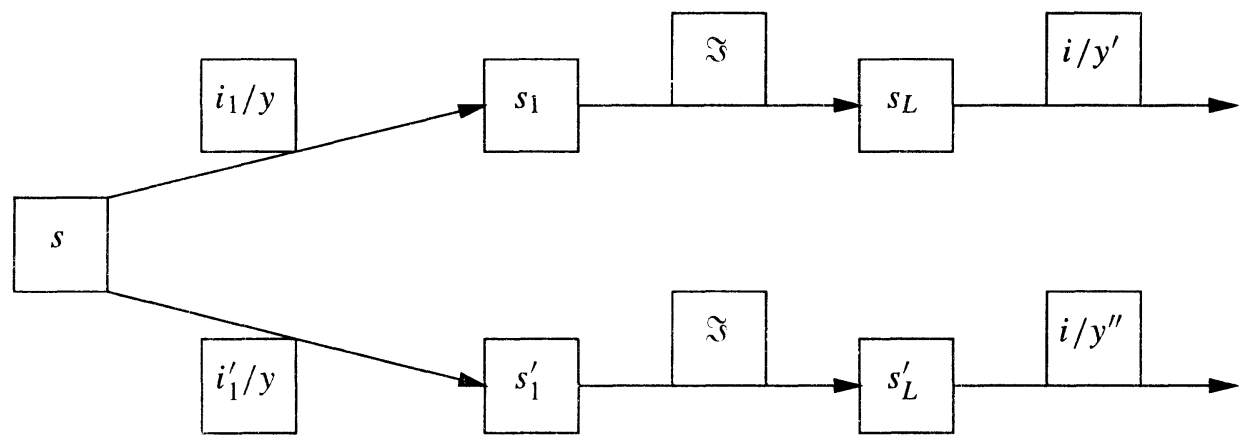

Определение 9. $K$-й приемник $\left(i, s_{L}, s_{L}^{\prime}\right) \quad B$-шестерки $\left(s, i_{1}, i_{1}^{\prime}, y, s_{1}, s_{1}^{\prime}\right)$ автомата $A$ $\left(B\right.$-семерки $\left.\left(s, i_{1}, i_{1}^{\prime}, y, y^{\prime}, s_{1}, s_{1}^{\prime}\right)\right)$ называется особым, если $\delta_{i} s_{L}=\delta_{i} s_{L}^{\prime}$.

В противном случае, $K$-й приемник $\left(i, s_{L}, s_{L}^{\prime}\right)$ называется неособым.

Теорема 2. Автоматное отображение $A_{s_{0}}$ увеличивает метрику $\rho$ не более, чем в $K$ раз $(K \geqslant 2)$, тогда и только тогда, когда для приведенного автомата

$$
A\left[s_{0}\right]=\left(I, S_{s_{0}}, O,\left(\delta_{i}\right)_{i \in I},\left(\beta_{i}\right)_{i \in I}\right)
$$

реализующего отображение $A_{s_{0}}$ выполнены условия:

- либо автомат $A\left[s_{0}\right]$ является внутренне автономным; 
- либо все $K$-приемники любой В-шестерки особые и все $(K-1)$-приемники любой В-семерки особые.

Конструктивность условий теорем 1, 2 будет показана позднее. Перейдем к их доказательствам.

Для доказательства теоремы 2 приведем две вспомогательные леммы.

Лемма 1. Отображение $\varphi: I^{*} \rightarrow O^{*}$ увеличивает метрику $\rho$ не более, чем в $K$ раз, тогда и только тогда, когда для любых слов $\mathfrak{\Im}, \Im^{\prime} \in I^{*}$ одинаковой длины из условия $\rho\left(\mathfrak{I}, \mathfrak{\Im}^{\prime}\right)=1$ следует, что

$$
\rho\left(\varphi(\mathfrak{\Im}), \varphi\left(\mathfrak{S}^{\prime}\right)\right) \leqslant K .
$$

Доказательство. Если отображение $\varphi$ увеличивает метрику не более, чем в $K$ раз, то про определению 1 для слов одинаковой длины справедлива импликация

$$
\rho\left(\mathfrak{I}, \mathfrak{I}^{\prime}\right)=1 \Longrightarrow \rho\left(\varphi(\mathfrak{s}), \varphi\left(\mathfrak{I}^{\prime}\right)\right) \leqslant K .
$$

Предположим теперь, что эта импликация верна. Рассмотрим слова $\mathfrak{I}_{0}, \mathfrak{I}_{m} \in I^{*}$ одинаковой длины, для которых $\rho\left(\mathfrak{\Im}_{0}, \mathfrak{\Im}_{m}\right)=m, m \geqslant 2$. Для слов $\Im_{0}, \mathfrak{\Im}_{m}$ найдутся слова $\mathfrak{\Im}_{1}, \mathfrak{\Im}_{2}, \ldots, \mathfrak{\Im}_{m-1} \in I^{*}$, при которых

$$
\rho\left(\mathfrak{I}_{0}, \mathfrak{\Im}_{1}\right)=\rho\left(\mathfrak{\Im}_{1}, \mathfrak{I}_{2}\right)=\ldots=\rho\left(\mathfrak{\Im}_{m-1}, \mathfrak{\Im}_{m}\right)=1 .
$$

Согласно импликации получаем, что

$$
\rho\left(\varphi\left(\mathfrak{\Im}_{0}\right), \varphi\left(\mathfrak{\Im}_{1}\right)\right) \leqslant K, \quad \rho\left(\varphi\left(\mathfrak{\Im}_{1}\right), \varphi\left(\mathfrak{\Im}_{2}\right)\right) \leqslant K, \ldots, \rho\left(\varphi\left(\mathfrak{\Im}_{m-1}\right), \varphi\left(\mathfrak{\Im}_{m}\right)\right) \leqslant K,
$$

откуда, согласно метрическим свойствам расстояния Хемминга следует, что

$$
\rho\left(\varphi\left(\mathfrak{\Im}_{0}\right), \varphi\left(\mathfrak{\Im}_{m}\right)\right) \leqslant m K .
$$

Лемма доказана.

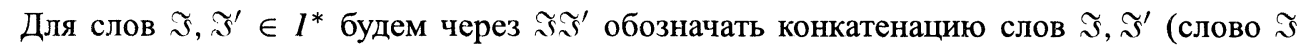
является началом слова $\left.\Im \Im^{\prime}\right)$.

Лемма 2. Если автоматное отображение $A_{s_{0}}$ увеличивает метрику $\rho$ не более, чем в $K$ раз, то при любом состоянии $s \in S_{s_{0}}$ автомата

$$
A\left[s_{0}\right]=\left(I, S_{s_{0}}, O,\left(\delta_{i}\right)_{i \in I},\left(\beta_{i}\right)_{i \in I}\right)
$$

автоматное отображение $A\left[s_{0}\right]_{s}=A_{s}$ увеличивает метрику не более, чем в $K$ раз.

Доказательство. Предположим противное. Пусть при выполнении условий леммы нашлось состояние $s \in S_{s_{0}}$, при котором отображение $A\left[s_{0}\right]_{s}$ не является увеличивающим метрику $\rho$ не более, чем в $K$ раз. Тогда найдутся $\mathfrak{I}^{\prime}, \mathfrak{I}^{\prime \prime} \in I^{*}$, при которых

$$
\left.\rho\left(A\left(s, \mathfrak{\Im}^{\prime}\right), A\left(s, \mathfrak{\Im}^{\prime \prime}\right)\right)>K \rho\left(\mathfrak{I}^{\prime}, \mathfrak{\Im}^{\prime \prime}\right)\right) \text {. }
$$

Из определения автомата $A\left[s_{0}\right]$ следует, что найдется слово $\Im \in I^{*}$, при котором $\delta \Im s_{0}=s$. Для конкатенаций $\mathfrak{s} \mathfrak{\Im}^{\prime}$ и $\mathfrak{\Im} \mathfrak{\Im}^{\prime \prime}$ слов $\mathfrak{\Im}, \mathfrak{\Im}^{\prime}, \mathfrak{\Im}^{\prime \prime}$ получаем неравенство

$$
\rho\left(A\left(s_{0}, \mathfrak{\Im} \mathfrak{\Im}^{\prime}\right), A\left(s_{0}, \mathfrak{s} \mathfrak{s}^{\prime \prime}\right)\right)>K \rho\left(\mathfrak{\Im} \mathfrak{s}^{\prime}, \mathfrak{\Im} \mathfrak{s}^{\prime \prime}\right),
$$

что противоречит условиям леммы. 
Доказательство теоремы 2. Пусть автоматное отображение $A_{s_{0}}$ увеличивает метрику $\rho$ не более, чем в $K$ раз, и автомат

$$
A\left[s_{0}\right]=\left(I, S_{s_{0}}, O,\left(\delta_{i}\right)_{i \in I},\left(\beta_{i}\right)_{i \in I}\right)
$$

не является внутренне автономным автоматом. Предположим, что в множестве всех $K$-приемников некоторой $B$-шестерки $\left(s, i_{1}, i_{1}^{\prime}, y_{1}, s_{1}, s_{1}^{\prime}\right)$ имеется неособенный $K$-приемник $\left(i_{L}, s_{L}, s_{L}^{\prime}\right)$, то есть в графе переходов автомата $A\left[s_{0}\right]$ существует диаграмма вида

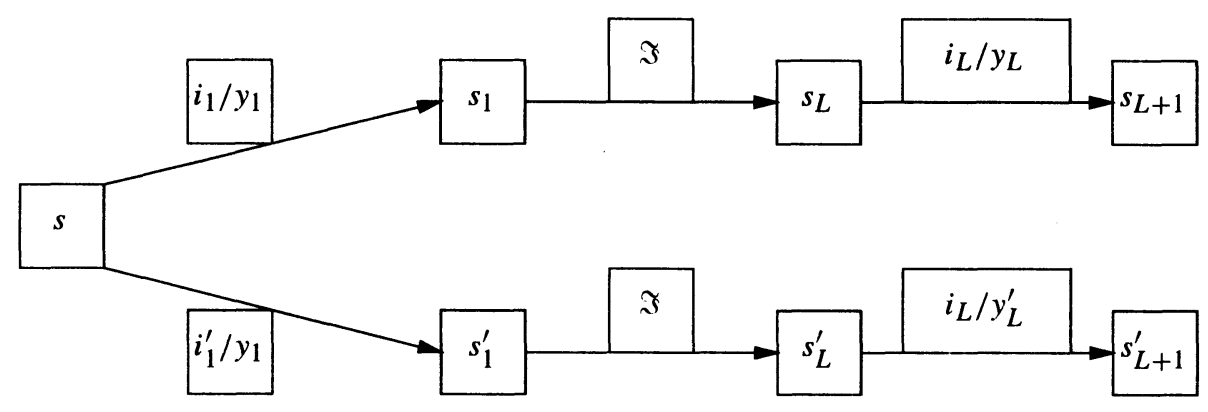

где

$i_{1} \neq i_{1}^{\prime}, \quad s_{1} \neq s_{1}^{\prime}, \quad s_{L} \neq s_{L}^{\prime}, \quad s_{L+1} \neq s_{L+1}^{\prime}, \quad y_{L} \neq y_{L}^{\prime}, \quad \rho\left(A\left(s_{1}, \Im i_{L}\right), A\left(s_{1}^{\prime}, \Im i_{L}\right)=K\right.$.

Так как $A\left[s_{0}\right]-$ приведенный автомат, для не равных состояний $s_{L+1}, s_{L+1}^{\prime}$ найдется слово $\hat{\Im} \in I^{*}$, при котором

$$
A\left(s_{L+1}, \hat{\Im}\right) \neq A\left(s_{L+1}^{\prime}, \hat{\Im}\right) .
$$

По этой причине

$$
\rho\left(A\left(s, i_{1} \Im i_{L} \hat{\Im}\right), A\left(s, i_{1}^{\prime} \Im i_{L} \hat{\Im}\right) \geqslant K+1\right.
$$

С другой стороны,

$$
\left.\left.\rho\left(i_{1} \Im i_{L} \hat{\Im}\right), i_{1}^{\prime} \Im i_{L} \hat{\Im}\right)\right)=1
$$

Следовательно, автоматное отображение $A_{s}$ не является отображением, увеличивающим метрику $\rho$ не более, чем в $K$ раз. Из леммы 2 вытекает, что и автоматное отображение $A_{s_{0}}$ не является отображением, увеличивающим метрику $\rho$ не более, чем в $K$ раз. Таким образом, получено противоречие с предположением о том, что в множестве всех $K$-приемников некоторой $B$-шестерки $\left(s, i_{1}, i_{1}^{\prime}, y_{1}, s_{1}, s_{1}^{\prime}\right)$ имеется неособенный $K$-приемник $\left(i_{L}, s_{L}, s_{L}^{\prime}\right)$. 
Аналогично получается противоречие, если предположить, что в множестве всех $(K-1)$-приемников некоторой $B$-семерки $\left(s, i_{1}, i_{1}^{\prime}, y, y^{\prime}, s_{1}, s_{1}^{\prime}\right)$ имеется неособенный $(K-1)$-приемник $\left(i_{L}, s_{L}, s_{L}^{\prime}\right)$, то есть в графе переходов автомата $A\left[s_{0}\right]$ существует диаграмма вида

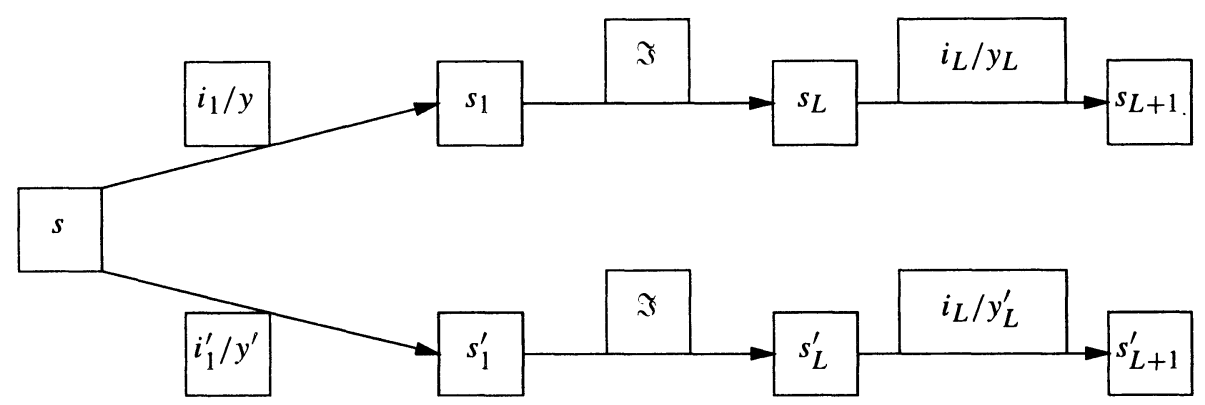

где

$$
\begin{gathered}
i_{1} \neq i_{1}^{\prime}, \quad y \neq y^{\prime}, \quad s_{1} \neq s_{1}^{\prime}, \quad s_{L} \neq s_{L}^{\prime}, \quad s_{L+1} \neq s_{L+1}^{\prime}, \quad y_{L} \neq y_{L}^{\prime}, \\
\rho\left(A\left(s_{1}, \Im i_{L}\right), A\left(s_{1}^{\prime}, \Im^{\prime} i_{L}\right)=K-1\right.
\end{gathered}
$$

В этом случае

$$
\rho\left(A\left(s, i_{1} \Im i_{L} \hat{\Im}\right), A\left(s, i_{1}^{\prime} \mathfrak{\Im} i_{L} \hat{\Im}\right) \geqslant K+1\right.
$$

а с другой стороны, $\left.\rho\left(i_{1} \Im i_{L} \hat{\Im}\right), i_{1}^{\prime} \Im i_{L} \hat{\Im}\right)=1$. Получаем противоречие.

Докажем теперь обратное утверждение теоремы 2.

Если автомат $A\left[s_{0}\right]$ внутренне автономен, то его отображение $A_{s_{0}}$ представимо в виде

$$
A_{s_{0}}\left(i_{1} i_{2} \ldots i_{k}\right)=\beta_{i_{1}} s_{0} \beta_{i_{2}} s_{1} \ldots \beta_{i_{k}} s_{k-1}
$$

для некоторых состояний $s_{1}, s_{2}, \ldots, s_{k-1}$ из $S$, не зависящих от $i_{1}, i_{2}, \ldots, i_{k}$. Поэтому отображение $A_{s_{0}}$ увеличивает $\rho$ не более, чем в один раз, и следовательно, не более, чем в $K$ раз при любом $K$. Предположим, что выполнены условия:

(1) автомат $A_{s_{0}}$ не является внутренне автономным,

(2) любой $K$-приемник (если он существует) каждой $B$-шестерки является особым,

(3) любой $(K-1)$-приемник (если он существует) каждой $B$-семерки является особым.

Из леммы 1 вытекает, что для доказательства достаточности условий теоремы 2 достаточно доказать, что из условия $\rho\left(\mathfrak{I}, \mathfrak{I}^{\prime}\right)=1$, $\mathfrak{\Im}, \mathfrak{\Im}^{\prime} \in I^{*}$ вытекает оценка

$$
\rho\left(A\left(s_{0}, \mathfrak{\Im}\right), A\left(s_{0}, \mathfrak{\Im}^{\prime}\right)\right) \leqslant K
$$

Докажем эту импликацию. Пусть $\rho\left(\mathfrak{s}, \mathfrak{I}^{\prime}\right)=1, \mathfrak{\Im}, \mathfrak{\Im}^{\prime} \in I^{*}$. Представим слова $\mathfrak{I}, \mathfrak{I}^{\prime}$ в виде $\mathfrak{\Im}=\mathfrak{I}_{1} i_{j} \mathfrak{I}_{2}, \mathfrak{\Im}^{\prime}=\mathfrak{I}_{1} i_{j}^{\prime} \mathfrak{\Im}_{2}$, где $i_{j} \neq i_{j}^{\prime}$. Априори возможны лишь следующие три случая, приведенные на рисунках. 
В первом случае $\delta_{i j} s_{j-1}=\delta_{i^{\prime} j} s_{j-1}=s_{j}$ :

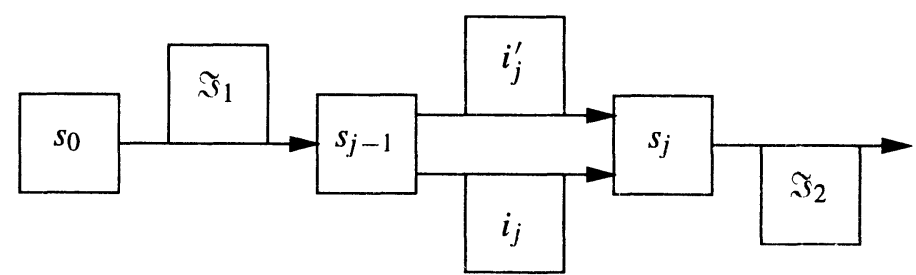

Во втором случае $\delta_{i j} s_{j-1} \neq \delta_{i^{\prime} j} s_{j-1}, \beta_{i j} s_{j-1}=\beta_{i^{\prime} j} s_{j-1}=y$ :

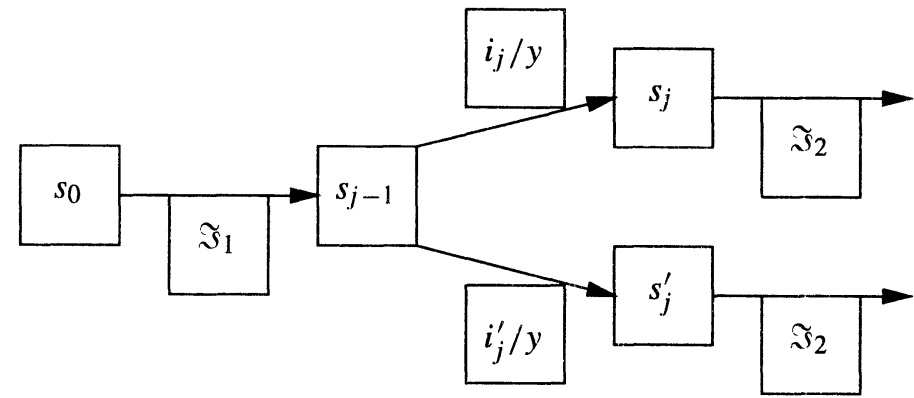

В третьем случае $\delta_{i j} s_{j-1} \neq \delta_{i^{\prime} j} s_{j-1}, \beta_{i j} s_{j-1}=y \neq \beta_{i^{\prime} j} s_{j-1}=y^{\prime}$ :

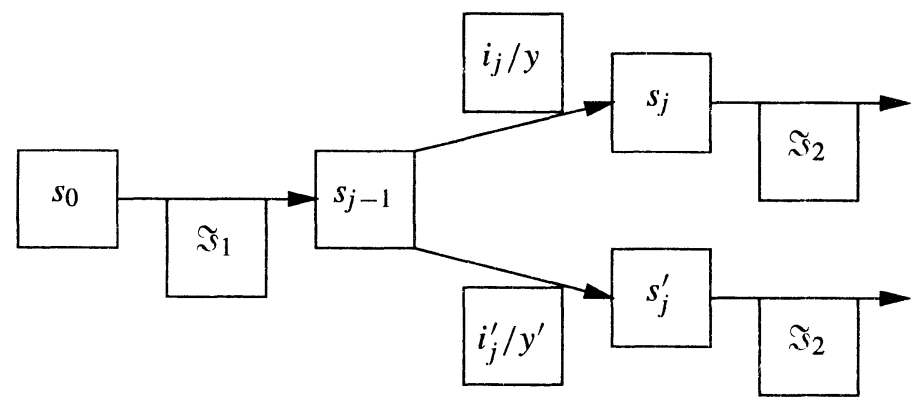

В первом случае очевидно, что

$$
\rho\left(A\left(s_{0}, \Im_{1} i_{j} \widetilde{\Im}_{2}\right), A\left(s_{0}, \Im_{1} i_{j}^{\prime} \Im_{2}\right)\right) \leqslant 1 \leqslant K .
$$

Рассмотрим оставшиеся два случая. Пусть имеет место второй случай. Предположим, что

$$
\rho\left(A\left(s_{0}, \widetilde{\Im}_{1} i_{j} \widetilde{\Im}_{2}\right), A\left(s_{0}, \widetilde{\Im}_{1} i_{j}^{\prime} \widetilde{\Im}_{2}\right)\right) \geqslant K+1
$$

Тогда

$$
\rho\left(A\left(s_{j}, \Im_{2}\right), A\left(s_{j}^{\prime}, \Im_{2}\right)\right) \geqslant K+1 .
$$

Рассмотрим номера позиций, на которых элементы последовательностей $A\left(s_{j}, \Im_{2}\right), A\left(s_{j}^{\prime}, \Im_{2}\right)$ различны. Пусть $y_{c}=\beta_{i} s_{c}, y_{c}^{\prime}=\beta_{i} s_{c}^{\prime}-K$-ые несовпадающие элементы в последовательностях $A\left(s_{j}, \mathfrak{\Im}_{2}\right), A\left(s_{j}^{\prime}, \mathfrak{\Im}_{2}\right)$. Тогда состояния $s_{c+1}, s_{c+1}^{\prime}$, отвечающие моменту времени $c+1$ функщионирования автомата $A$, начинающегося с состояний $s_{j}, s_{j}^{\prime}$, при входной последовательности $\Im_{2}$ не совпадают, так как в последовательностях $A\left(s_{j}, \Im_{2}\right), A\left(s_{j}^{\prime}, \Im_{2}\right)$ имеется $(K+1)$-я позиция несовпадения их элементов. Таким образом, доказано существование неособенного $K$-приемника $B$-шестерки 
$\left(s_{j-1}, i_{j}, i_{j}^{\prime}, y, s_{j}, s_{j}^{\prime}\right)$, что противоречит условию (2). Итак, для второго случая доказано неравенство

$$
\rho\left(A\left(s_{0}, \Im_{1} i_{j} \Im_{2}\right), A\left(s_{0}, \Im_{1} i_{j}^{\prime} \Im_{2}\right)\right) \leqslant K .
$$

Для третьего случая это неравенство доказывается аналогично.

Доказательство теоремы 1. Для автомата $A\left[s_{0}\right]$ априори возможны два случая:

(1) автомат $A\left[s_{0}\right]$ внутренне автономен, что равносильно тому, что множество всех $B$-шестерок и множество всех $B$-семерок являются пустыми множествами,

(2) хотя бы одно из указанных множеств не являются пустым, то есть автомат $A\left[s_{0}\right]$ не является автономным.

Предположим, что автоматное отображение $A_{s_{0}}$ не увеличивает метрику $\rho$. Если в автомате $A\left[s_{0}\right]$ имеется $B$-семерка $\left(s, i, i^{\prime}, y, y^{\prime}, s_{1}, s_{1}^{\prime}\right)$, то в его графе переходов имеется диаграмма вида

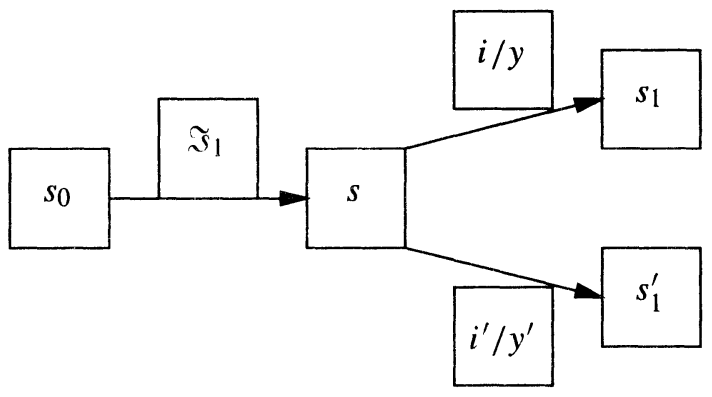

где $i \neq i^{\prime}, y \neq y^{\prime}, s_{1} \neq s_{1}^{\prime}$.

Так как $A\left[s_{0}\right]$ - приведенный автомат, найдется слово $\mathfrak{I}_{2} \in I^{*}$, при котором $A\left(s_{1}, \mathfrak{\Im}_{2}\right) \neq A\left(s_{2}, \mathfrak{\Im}_{2}\right)$. Тогда

$$
\rho\left(\mathfrak{\Im}_{1} i \widetilde{\Im}_{2}, \mathfrak{\Im}_{1} i^{\prime} \mathfrak{\Im}_{2}\right)=1, \quad \rho\left(A\left(s_{0}, \mathfrak{\Im}_{1} i \mathfrak{\Im}_{2}\right), A\left(s_{0}, \mathfrak{\Im}_{1} i^{\prime} \mathfrak{\Im}_{2}\right)\right) \geqslant 2,
$$

что противоречит предположению о том, что автоматное отображение $A_{s_{0}}$ не увеличивает метрику $\rho$. Таким образом, если автоматное отображение $A_{s_{0}}$ не увеличивает метрику $\rho$, то в автомате $A\left[s_{0}\right]$ отсутствуют $B$-семерки.

Пусть отображение $A_{s_{0}}$ не увеличивает метрику $\rho$ и автомат $A\left[s_{0}\right]$ не является внутренне автономным. Предположим, что в множестве всех приемников некоторой $B$-шестерки $\left(s, i, i^{\prime}, y, s_{1}, s_{2}\right)$ имеется неособый приемник, то есть в графе переходов автомата $A\left[s_{0}\right]$ имеется диаграмма вида

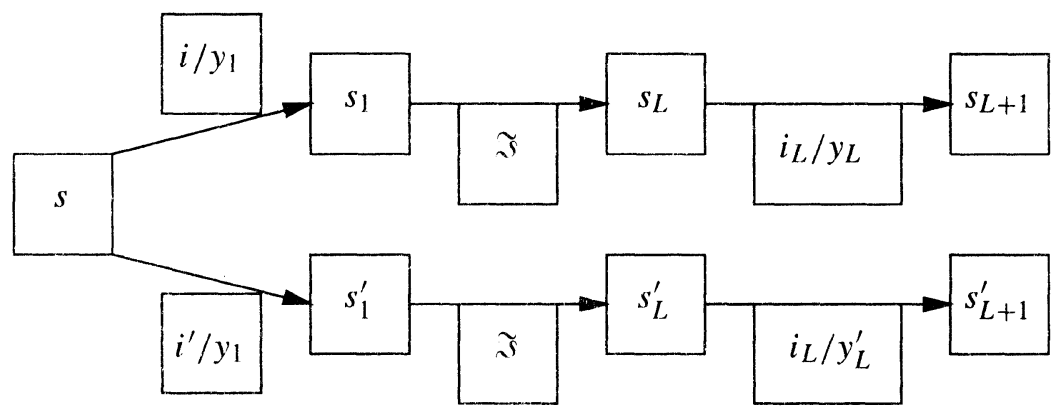


где $s_{1} \neq s_{1}^{\prime}, i \neq i^{\prime}, \Im \in I^{*}, y_{L} \neq y_{L}^{\prime}, s_{L+1} \neq s_{L+1}^{\prime}$.

Из приведенности автомата $A\left[s_{0}\right]$ следует существование слова $\hat{\Im} \in I^{*}$, при котором

$$
A\left(s_{L+1}, \hat{\Im}\right) \neq A\left(s_{L+1}^{\prime}, \hat{\Im}\right)
$$

Тогда

$$
\rho\left(i \Im i_{L} \hat{\Im}, i^{\prime} \Im i_{L} \hat{\Im}\right)=1, \quad \rho\left(A\left(s, i \Im i_{L} \hat{\Im}\right), A\left(s, i^{\prime} \Im i_{L} \hat{\Im}\right)\right) \geqslant 2 .
$$

Следовательно, отображение $A_{s}$ не является отображением, не увеличивающим $\rho$. Тогда по лемме 2 и отображение $A_{s_{0}}$ не является отображением, не увеличивающим $\rho$, что противоречит начальному предположению. Таким образом, доказано, что если автомат $A\left[s_{0}\right]$ не является внутренне автономным и у него отсутствуют $B$-семерки и отображение $A_{s} 0$ не увеличивает $\rho$, то каждый приемник любой $B$-шестерки является особым.

Перейдем теперь к доказательству достаточности условий теоремы 1 . Очевидно, что при внутренне автономном автомате $A\left[s_{0}\right]$ автоматное отображение $A_{s_{0}}$ не увеличивает метрику $\rho$.

Предположим, что $A\left[s_{0}\right]$ не является внутренне автономным автоматом, в нем отсутствуют $B$-семерки и каждый приемник любой $B$-шестерки особый. Согласно лемме 1 для доказательства того, что отображение $A_{s_{0}}$ не увеличивает $\rho$, достаточно доказать, что выполняется импликация

$$
\left\{\rho\left(\mathfrak{I}, \mathfrak{I}^{\prime}\right)=1, \mathfrak{\Im}, \mathfrak{\Im}^{\prime} \in I^{*}\right\} \Longrightarrow\left\{\rho\left(A\left(s_{0}, \mathfrak{I}\right), A\left(s_{0}, \mathfrak{I}^{\prime}\right) \leqslant 1\right\} .\right.
$$

Пусть $\rho\left(\mathfrak{I}, \mathfrak{I}^{\prime}\right)=1$ для некоторых $\mathfrak{\Im}, \mathfrak{I}^{\prime} \in I^{*}$. Представим $\mathfrak{\Im}, \mathfrak{I}^{\prime}$ в виде

$$
\mathfrak{\Im}=\mathfrak{\Im}_{1} i_{j} \mathfrak{\Im}_{2}, \quad \Im^{\prime}=\mathfrak{I}_{1} i_{j}^{\prime} \mathfrak{\Im}_{2},
$$

где $i_{j} \neq i_{j}^{\prime}, \Im_{1}, \Im_{2} \in \Im^{*}$. Рассмотрим пути в графе переходов автомата $A\left[s_{0}\right]$, отвечающие состоянию $s_{0}$ и входным словам $\mathfrak{\Im}, \mathfrak{\Im}^{\prime}$. Априори возможны лишь следующие случаи.

В первом случае

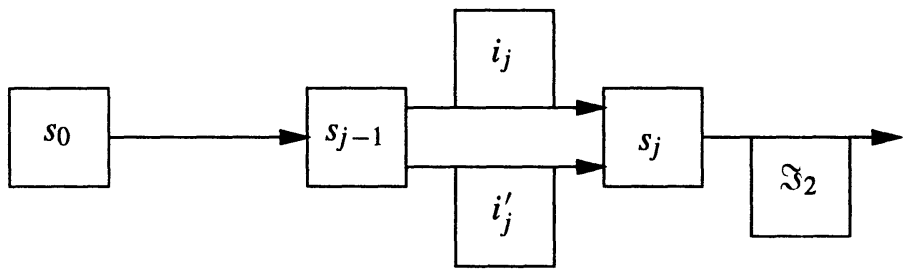

Во втором случае, где $s_{j} \neq s_{j}^{\prime}$,

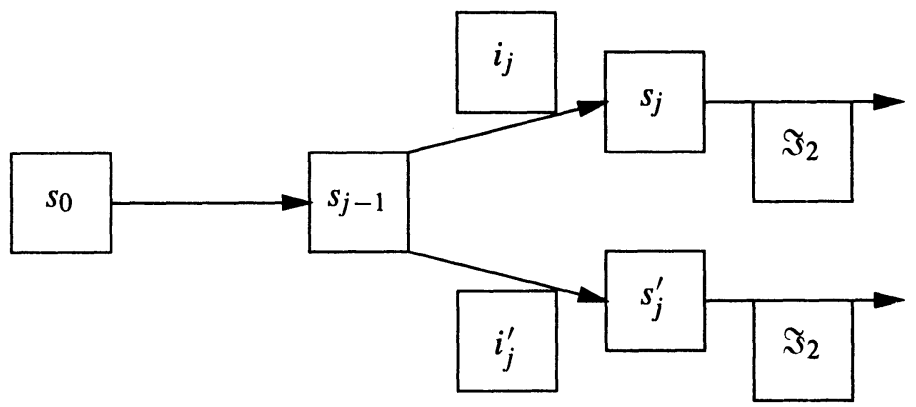


Второй случай распадается на два подслучая:

$$
\beta_{i j} s_{j-1}=\beta_{i^{\prime}, j} s_{j-1}, \quad \beta_{i j} s_{j-1} \neq \beta_{i^{\prime} j} s_{j-1} .
$$

В первом подслучае, используя условие, что каждый приемник любой $B$-шестерки является особым, заключаем, что

$$
\rho\left(A\left(s_{0}, \mathfrak{\Im}_{1} i_{j} \mathfrak{s}_{2}\right), A\left(s_{0}, \Im_{1} i_{j}^{\prime} \Im_{2}\right)\right) \leqslant 1 .
$$

Второй подслучай невозможен, так как по условию у автомата $A\left[s_{0}\right]$ отсутствуют $B$-семерки. Таким образом, доказана достаточность условий теоремы 1.

Напомним, что автомат $A=\left(I, S, O,\left(\delta_{1}\right)_{i \in I},\left(\beta_{i}\right)_{i \in I}\right)$ называется перестановочным, если его частичные функции перехода $\left(\delta_{i}\right)_{i \in I}$ осуществляют биекции $S$ в $S$.

В классе автоматных отображений выделим два подкласса: множество всех инъективных автоматных отображений $I^{*}$ в $O^{*}$ и множество всех автоматных отображений $A_{s}$, для которых автомат $A[s]$ является перестановочным.

Для этих подклассов автоматных отображений приведем некоторые следствия доказанных теорем 1 и 2 . Для автомата

$$
A\left[s_{0}\right]=\left(I, S_{s_{0}}, O,\left(\delta_{i}\right)_{i \in I},\left(\beta_{i}\right)_{i \in I}\right)
$$

через $\beta_{s}, s \in S$, обозначим отображение $I$ в $O$, задаваемое равенствами $\beta_{s}(i)=\beta_{i}(s)$, $i \in I$. Очевидно следующее утверждение.

Предложение 1. Автоматное отображение $A_{s_{0}}$ инъективно тогда и только тогда, когда все отображения $\beta_{s}, s \in S_{s_{0}}$, инъективны.

Отметим, что наличие $B$-шестерки у автомата $A_{s_{0}}$ является достаточным условием не инъективности отображения $A_{s_{0}}$. Из этого факта, предложения 1 и теоремы 1 вытекает следующее утверждение.

Следствие 1. Пусть $A_{s_{0}}-$ инъективное отображение и $A\left[s_{0}\right]-$ приведенный автомат. Отображение $A_{s_{0}}$ не увеличивает метрику $\rho$ тогда и только тогда, когда автомат $A\left[s_{0}\right]$ является внутренне автономным.

Из теоремы 1 и определения перестановочности автомата $A$ вытекает следующее утверждение.

Следствие 2. Пусть $A\left[s_{0}\right]$ - приведенный перестановочный автомат. Отображение $A_{s_{0}}$ не увеличивает метрику $\rho$ тогда и только тогда, когда автомат $A\left[s_{0}\right]$ является внутренне автономным.

Из теоремы 2 несложно получить следующие следствия.

Следствие 3. Пусть $A_{s_{0}}-$ инъективное отображение и $A\left[s_{0}\right]-$ приведенный автомат. Отображение $A_{s_{0}}$ увеличивает метрику $\rho$ не более, чем в $K$ раз, $K \leqslant 2$, тогда $u$ только тогда, когда либо автомат $A\left[s_{0}\right]$ является внутренне автономным, либо все его $(K-1)$-приемники любой В-семерки являются особыми.

Следствие 4. Пусть $A\left[s_{0}\right]$ - приведенный перестановочный автомат. Отображение $A_{s_{0}}$ увеличивает метрику $\rho$ не более, чем в $K$ раз, $K \geqslant 2$, тогда и только тогда, когда автомат $A\left[s_{0}\right]$ является внутренне автономным. 
Отметим тот факт, что расширения класса автоматных отображений, осуществляемых инициальными приведенными перестановочными автоматами, увеличивающих метрику $\rho$ не более, чем в один раз, при переходе к автоматным отображениям, увеличивающим метрику $\rho$ строго более, чем в один раз, не происходит.

Замечание 1. Для проверки условий теорем 1, 2 требуется проверить наличие или отсутствие в графе переходов автомата $A\left[s_{0}\right]$ некоторых диаграмм (фрагментов). Сложность такой проверки выражается через максимальную длину входных слов, характеризующих диаграмму. Несложно доказать, что для проверки приемника $B$-шестерки на его особенность достаточно рассматривать входные слова $\mathfrak{I}$ (см. определение 5) длины, не превосходящей

$$
M=\frac{|S|(|S|-1)}{2}-1
$$

Для проверки $K$-приемника $B$-шестерки на его особенность достаточно рассматривать входные слова $\mathfrak{s}$ (см. определение 9) длины, не превосходящей $K M$. Аналогично, для $(K-1)$-приемника $B$-семерки - слова длины не более, чем $(K-1) M$. Получение минимальных оценок длин указанных слов $\Im$ представляет собой отдельную задачу. Приведенные оценки доказывают лишь конструктивность условий теорем 1 и 2.

\section{4. Описание множества $A G\left(I^{*}, O^{*}, \varepsilon, K\right)$}

Предположим, что некоторое автоматное отображение $A_{s_{0}}: I^{*} \rightarrow O^{*}$ увеличивает бинарное отношение $\varepsilon$ не более, чем в $K$ раз, то есть для любых $\mathfrak{s}, \mathfrak{s}^{\prime} \in I^{*}$ и любого $n \in\{1, \ldots,|\Im|\}$ имеется число $m \in\{1, \ldots, K n\}$ такое, что выполняется импликация

$$
\mathfrak{\Im} \varepsilon^{n} \mathfrak{\Im}^{\prime} \Longrightarrow A\left(s_{0}, \mathfrak{\Im}\right) \varepsilon^{m} A\left(s_{0}, \Im^{\prime}\right) \text {. }
$$

Так как автоматное отображение сохраняет длины слов, из этого соотношения следует, что $m=n$. В частности, $A_{s_{0}}$ не размножает искажений типа пропуска букв. Обратно, если $A_{s_{0}}$ не размножает искажения типа пропуска букв, то, очевидно, $A_{s_{0}}$ Увеличивает бинарное отношение $\varepsilon$ не более, чем в $K$ раз, при любом $K$. Таким образом, множество $A G\left(I^{*}, O^{*} \varepsilon, K\right)$ всех автоматных инъективных отображений $A_{s}: I^{*} \rightarrow O^{*}$, увеличивающих бинарное отношение $\varepsilon$ не более, чем в $K$ раз, совпадает с множеством $A G\left(I^{*}, O^{*}, \varepsilon, 1\right)$. Описание последнего множества дается в следующей теореме.

Теорема 3. Инъективное автоматное отображение $A_{s_{0}}$ не размножает искажения типа пропуска букв тогда и только тогда, когда для автомата

$$
A\left[s_{0}\right]=\left(I, S_{\left.s_{0}\right)}, O,\left(\delta_{i}\right)_{i \in I},\left(\beta_{i}\right)_{i \in I}\right)
$$

при любом $s \in S_{s_{0}}$ отображение $\beta_{s}$ не зависит от выбора $s \in S_{s_{0}}$.

Отметим, что данное утверждение, по-видимому можно доказать, используя результаты работ М. М. Глухова $[4,5]$ по описанию инъективных отображений, не распространяющих искажения. Ниже дается теоретико-автоматное доказательство теоремы 3.

Приведем вспомогательные утверждения, представляющие и самостоятельный интерес. Следующее утверждение очевидно. 
Предложение 2. Инъективное отображение $\varphi: I^{*} \rightarrow O^{*}$ не размножает искажения типа пропуска букв тогда и только тогда, когда из $\mathfrak{s} \varepsilon \mathfrak{I}^{\prime}, \mathfrak{\Im}, \mathfrak{I}^{\prime} \in I^{*}$, следует, что $\varphi(\mathfrak{I}) \varepsilon \varphi\left(\mathfrak{\Im}^{\prime}\right)$.

Пусть $A\left[s_{0}\right]=\left(I, S_{s_{0}}, O,\left(\delta_{i}\right)_{i \in I},\left(\beta_{i}\right)_{i \in I}\right)-$ автомат, реализующий автоматное отображение $A_{s_{0}}$. Ниже мы допускаем, что автомат $A\left[s_{0}\right]$ может быть и неприведенным автоматом.

Предложение 3. Если автоматное отображение $A_{s_{0}}$ не размножает искажения типа пропуска букв, то при любом $s \in S_{s_{0}}$ автоматное отображение $A_{s}$ не размножает искажения типа пропуска букв.

Доказательство. Пусть для $s \in S_{s_{0}}$ автоматное отображение $A_{s}$ не размножает искажения типа пропуска букв. Тогда при любых $i \in I, \mathfrak{s} \in I^{*}, \mathfrak{s} \neq \varnothing$ и $\mathfrak{\Im} \neq \mathfrak{\Im}^{\prime}$, для которого $\mathfrak{s} \mathfrak{\Im}^{\prime}$, справедливы отношения $i \Im \varepsilon i \Im^{\prime}$ и $A(s, i \Im) \varepsilon A\left(s, i \Im^{\prime}\right)$. Учитывая, что первые символы в словах $A(s, i \mathfrak{\Im}), A\left(s, i \mathfrak{\Im}^{\prime}\right)$ равны, получаем, что

$$
A\left(\delta_{i} s, \mathfrak{\Im}\right) \varepsilon A\left(\delta_{i} s, \Im^{\prime}\right),
$$

то есть (см. предложение 2) отображение $A_{s^{\prime}}$, задаваемое равенствами $s^{\prime}=\delta_{i} s$, не размножает искажения типа пропуска букв. Следовательно, при любом $s \in S_{s_{0}}$ автоматное отображение $A_{s}$ не размножает искажения типа пропуска букв.

Доказательство теоремы 3. Пусть инъективное отображение $A_{s_{0}}$ не размножает искажения типа пропуска букв. Тогда (см. предложение 3) при любом $s \in S_{s 0}$ автоматное отображение $A_{s}$ не размножает искажения типа пропуска букв. При этом отображение $A_{s}$ инъективно (см. предложение 1).

Покажем, что при любых различных $i_{1}, i_{2} \in I$ выходное слово $A\left(s, i_{1}, i_{2}\right)=y_{1} y_{2}$ таково, что $y_{1} \neq y_{2}$. Предположим, что $A\left(s, i_{1}, i_{2}\right)=y y$. Тогда $i_{1} i_{2} \varepsilon i_{2}$, yy $\varepsilon A\left(s, i_{2}\right)$. Отсюда получаем, что $A\left(s, i_{2}\right)=y$, что противоречит инъективности отображения $A_{s}$, так как $A\left(s, i_{1}\right)=y$.

Итак, при любом $i \in I, i \neq i^{\prime}$,

$$
i i^{\prime} \varepsilon i^{\prime}, \quad A\left(s, i i^{\prime}\right)=y y^{\prime},
$$

где $y \neq y^{\prime}$. Откуда получаем, что $y y^{\prime} \varepsilon A\left(s, i^{\prime}\right), A(s, i)=y$. Следовательно, $\beta_{s}\left(i^{\prime}\right)=y^{\prime}$, a из $A\left(s, i i^{\prime}\right)=y y^{\prime}$ следует, что $\beta_{s_{1}}\left(i^{\prime}\right)=y^{\prime}, s_{1}=\delta_{i} s$. Поэтому

$$
\beta_{s_{1}}\left(i^{\prime}\right)=y^{\prime}=\beta_{s}\left(i^{\prime}\right)
$$

для всех $i^{\prime}$ из $I$, не равных $i$. Так как любое отображение $A_{s^{\prime}}, s^{\prime} \in S_{s_{0}}$, не размножает искажения типа пропуска букв, для любых $i \in I, s^{\prime} \in S_{s_{0}}$ получаем, что

$$
\beta_{s_{1}^{\prime}}\left(i^{\prime}\right)=\beta_{s^{\prime}}\left(i^{\prime}\right), \quad s_{1}^{\prime}=\delta_{i} s^{\prime}
$$

для всех $i^{\prime} \neq i$.

Покажем теперь, что эти равенства выполняются и при $i^{\prime}=i$. Очевидно, для этого достаточно доказать, что $\beta_{s_{1}}(i)=\beta_{s}(i), s_{1}=\delta_{i} s$, при любом $i \in I$. Для доказательства последних равенств, в свою очередь, достаточно доказать, что при любом $i \in I$

$$
A(s, i i)=y y
$$


при некотором $y \in O$, зависящем от $i$.

Предположим, что нашлось $i \in I$, при котором $A(s, i i)=y_{1} y_{2}, y_{1} \neq y_{2}$. Тогда при некотором $i^{\prime} \in I, i \neq i^{\prime}$, получаем, что

$$
A\left(s, i i i^{\prime}\right)=y_{1} y_{2} y^{\prime}
$$

при некотором $y^{\prime} \in O$. Используя равенства (3), находим, что

$$
\beta_{s}\left(i^{\prime}\right)=\beta_{s_{1}}\left(i^{\prime}\right)=\beta_{s_{2}}\left(i^{\prime}\right)=y^{\prime}, \quad s_{1}=\delta_{i} s, \quad s_{2}=\delta_{i} \delta_{i} s .
$$

Следовательно, учитывая инъективность отображения, получаем, что $y^{\prime} \neq y_{1}, y^{\prime} \neq y_{2}$. Если $|O|=2$, то получено противоречие, и следовательно, доказано, что $y_{1}=y_{2}$, то есть доказана необходимость условий теоремы 2.

Пусть $|O| \geqslant 3$. Нами доказано, что элементы $y_{1}, y_{2}, y_{3}=y^{\prime}$ различны. Справедливы равенства

$$
A\left(s, i i i^{\prime}\right)=y_{1} y_{2} y_{3}, \quad \beta_{s}\left(i^{\prime}\right)=\beta_{s_{1}}\left(i^{\prime}\right)=\beta_{s_{2}}\left(i^{\prime}\right)=y^{\prime}, \quad s_{1}=\delta_{i} s, \quad s_{2}=\delta_{i} \delta_{i} s .
$$

Из этих равенств получаем, что

$$
\begin{aligned}
A\left(s, i i^{\prime}\right) & =y_{1} y_{3}, & & \\
A\left(s, i i^{\prime} i\right) & =y_{1} y_{3}\left(\beta_{i} \delta_{i^{\prime}} \delta_{i} s\right)=y_{1} y_{3} \beta_{\delta i^{\prime} \delta i s}(i)=y_{1} y_{3} \beta_{s_{1}}(i)=y_{1} y_{3} y_{2}, & & s_{1}=\delta_{i} s, \\
A\left(s, i^{\prime} i\right) & =y_{3}\left(\beta_{i} \delta_{i^{\prime}} s\right)=y_{3} \beta_{s_{1}^{\prime}}(i)=y_{3} \beta_{s}(i)=y_{3} y_{1}, & & s_{1}^{\prime}=\delta_{i}^{\prime} s .
\end{aligned}
$$

Из отношения $i i^{\prime} i \varepsilon i^{\prime} i$ следует отношение

$$
A\left(s, i i^{\prime} i\right) \varepsilon A\left(s, i^{\prime} i\right)
$$

однако слово $A\left(s, i i^{\prime} i\right)=y_{1} y_{3} y_{2}$ не находится в бинарном отношении $\varepsilon$ со словом $A\left(s, i^{\prime} i\right)=y_{3} y_{1}$. Полученное противоречие завершает доказательство промежуточного утверждения о том, что $y_{1}=y_{2}$, которое, как раньше было показано, достаточно для завершения доказательства необходимости условий теоремы 3. Достаточность ее условий очевидна.

Следствие 5. Если инъективное отображение $A_{s_{0}}$ не размножает искажений типа пропуска букв и $A\left[s_{0}\right]=\left(I, S_{s_{0}}, O,\left(\delta_{i}\right)_{i \in I},\left(\beta_{i}\right)_{i \in I}\right)-$ приведенный автомат, то $\left|S_{s_{0}}\right|=1$.

\section{5. Описание множества $A G\left(I^{*}, O^{*}, D, K\right)$}

Обозначим $D$ метрику Левенштейна [3] на множестве $I^{*}\left(O^{*}\right)$, пусть $D\left(\mathfrak{I}, \mathfrak{\Im}^{\prime}\right)$ означает минимальное число выпадений и вставок букв для получения из слова $\mathfrak{\Im}$ слова $\mathfrak{\Im}^{\prime}$.

Определение 10. Отображение $\varphi: I^{*} \rightarrow O^{*}$ называется отображением, увеличивающим метрику $D$ не более, чем в $K$ раз, если для любых слов $\mathfrak{s}, \Im^{\prime} \in I^{*}$ выполняется условие

$$
K D\left(\mathfrak{\Im}, \mathfrak{\Im}^{\prime}\right) \geqslant D\left(\varphi(\mathfrak{I}), \varphi\left(\mathfrak{I}^{\prime}\right)\right) .
$$

Через $A G\left(I^{*}, O^{*} D, K\right)$ обозначим множество всех автоматных инъективных отображений $A_{s}: I^{*} \rightarrow O^{*}$, увеличивающих метрику $D$ не более, чем в $K$ раз. 
Предположим, что некоторое автоматное отображение $A_{s_{0}}: I^{*} \rightarrow O^{*}$ увеличивает метрику $D$ не более, чем в $K$ раз, то есть для любых слов $\mathfrak{I}, \Im^{\prime} \in I^{*}$ выполняется условие

$$
K D\left(\mathfrak{I}, \mathfrak{\Im}^{\prime}\right) \geqslant D\left(A_{s_{0}}(\mathfrak{s}), A_{s_{0}}\left(\mathfrak{\Im}^{\prime}\right)\right)
$$

Следующая лемма доказывается так же, как лемма 1.

Лемма 3. Отображение $\varphi: I^{*} \rightarrow O^{*}$ увеличивает метрику $D$ не более, чем в $K$ раз, тогда и только тогда, когда для любых слов $\mathfrak{\Im}, \mathfrak{\Im}^{\prime} \in I^{*}$ из условия $D\left(\mathfrak{I}, \mathfrak{I}^{\prime}\right)=1$ следует, чmo

$$
D\left(\varphi(\mathfrak{\Im}), \varphi\left(\mathfrak{\Im}^{\prime}\right)\right) \leqslant K
$$

Ниже дается описание множества $A G\left(I^{*}, O^{*} D, K\right)$ для $K=1$ и $K=2$. Случай $K \geqslant 3$ остается открытым.

Предложение 4. Автоматное отображение $A_{s_{0}}: I^{*} \rightarrow O^{*}$ увеличивает метрику $D$ не более, чем в 1 раз, тогда и только тогда, когда оно увеличивает бинарное отночение $\varepsilon$ не более, чем в 1 раз.

Доказательство. Предположим, что автоматное отображение $A_{s_{0}}: I^{*} \rightarrow O^{*}$ увеличивает метрику $D$ не более, чем в 1 раз. Пусть для слов $\mathfrak{F}, \mathfrak{I}^{\prime}$ выполняется равенство $D\left(\mathfrak{I}, \mathfrak{I}^{\prime}\right)=1$, то есть либо $\mathfrak{乛} \varepsilon \mathfrak{I}^{\prime}$, либо $\mathfrak{I}^{\prime} \varepsilon \mathfrak{\Im}$. Так как автоматное отображение сохраняет длины слов, то в первом случае из неравенства $D\left(A_{s 0}(\mathfrak{I}), A_{s 0}\left(\mathfrak{I}^{\prime}\right)\right) \leqslant 1$ получаем, что $A_{s_{0}}(\mathfrak{\Im}) \varepsilon A_{s_{0}}\left(\mathfrak{\Im}^{\prime}\right)$. Во втором случае получаем, что $A_{s_{0}}\left(\mathfrak{S}^{\prime}\right) \varepsilon A_{s_{0}}(\mathfrak{I})$. Следовательно (см. лемму 3), если отображение $A_{s 0}: I^{*} \rightarrow O^{*}$ увеличивает метрику $D$ не более, чем в 1 раз, то оно увеличивает бинарное отношение $\varepsilon$ не более, чем в 1 раз. Обратное утверждение очевидно.

Предложение 5. Автоматное отображение $A_{s_{0}}: I^{*} \rightarrow O^{*}$ увеличивает метрику $D$ не более, чем в 2 раза, тогда и только тогда, когда оно увеличивает бинарное отночение $\varepsilon$ не более, чем в 1 раз.

Доказательство. Предположим, что автоматное отображение $A_{s_{0}}: I^{*} \rightarrow O^{*}$ увеличивает метрику $D$ не более, чем в 2 раза. Пусть для слов $\mathfrak{I}, \mathfrak{F}^{\prime}$ выполняется равенство $D\left(\mathfrak{I}, \mathfrak{I}^{\prime}\right)=1$, то есть либо $\mathfrak{s} \varepsilon \mathfrak{I}^{\prime}$, либо $\mathfrak{\Im}^{\prime} \varepsilon \mathfrak{\Im}$. Так как автоматное отображение сохраняет длины слов, то в первом случае из неравенства $D\left(A_{s_{0}}(\mathfrak{I}), A_{s_{0}}\left(\mathfrak{\Im}^{\prime}\right)\right) \leqslant 2$ получаем, что $A_{s_{0}}(\Im) \varepsilon A_{s_{0}}\left(\Im^{\prime}\right)$. Во втором случае получаем, что $A_{s_{0}}\left(\Im^{\prime}\right) \varepsilon A_{s_{0}}(\Im)$. Следовательно (см. лемму 3 ), если отображение $A_{s 0}: I^{*} \rightarrow O^{*}$ увеличивает метрику $D$ не более, чем в 2 раза, то оно увеличивает бинарное отношение $\varepsilon$ не более, чем в 1 раз. Обратное утверждение очевидно.

Таким образом, множества $A G\left(I^{*}, O^{*}, D, 1\right)$ и $A G\left(I^{*}, O^{*}, D, 2\right)$ совпадают с множеством автоматных инъективных отображений, сохраняющих бинарное отношение $\varepsilon$ (см. теорему 3).

Автор благодарен С. В. Полину за полезные замечания, позволившие улучшить качество работы. 


\section{Список литературы}

1. Бабаш А. В., Глухов М. М., Шанкин Г. П., О преобразованиях множества слоев в конечном алфавите, не размножающих искажений. Дискретная математика (1997) 9, №3, 3-19.

2. Глухов М. М., Инъективные отображения слов, не размножающие искажений типа пропуска букв. Дискретная математика (1999) 11, №2, 20-39.

3. Левенштейн В. И., О совершенных кодах в метрике выпадений и вставок. Дискретная математика (1991) 3, №1, 3-20.

4. Глухов М. М., Инъективные отображения слов, не размножающие искажений. Maтем. вопросы киберн. (1998) 7, 349-350.

5. Глухов М. М., Инъективные отображения слов, не размножающие искажений. Tpyды по дискретной математике (2001) 4, 17-32.

Статья поступила 18.09.2001. 